AIAA-2000-4244

\title{
ON-ORBIT CALIBRATION OF SATELLITE GYROSCOPES ${ }^{* \dagger}$
}

\author{
Joseph A. Hashmall, Mark Radomski, and Joseph Sedlak \\ Computer Sciences Corporation, 7700 Hubble Drive, Lanham/Seabrook, MD 20706 \\ Phone: (301)-794-2175, e-mail: jhashmal@csc.com
}

\begin{abstract}
In order to maneuver satellites accurately from one attitude to another, onboard rate sensing gyroscopes usually must be calibrated after launch. Several algorithms have been used to determine gyro biases, misalignments, and scale factors. This paper describes algorithms that have been used in the past, discusses their advantages and limitations, and describes a new algorithm and the gyro calibration results obtained using this new algorithm. The new algorithm has significant operational advantages in addition to being at least as accurate as other algorithms.
\end{abstract}

\section{INTRODUCTION}

Knowledge of spacecraft angular rates is essential in any attitude determination method that uses data from different times, as well as in controlling attitude maneuvers. On most three-axis stabilized spacecraft these rates are measured using gyroscopes-also known as Inertial Reference Units (IRU).

In order to use gyro measurements they must be converted from raw measurements in the sensor frame to angular measurements in the body frame. Considering three independent axes of gyro data at a time, this can be accomplished by:

$$
\vec{\omega}=G\left(\vec{\omega}_{o}-\vec{b}\right)
$$

where $\bar{\omega}_{o}$ is the observed rate vector, $\bar{b}$ is a bias vector in the sensor frame and $\boldsymbol{G}$ a matrix that scales the rates and rotates them into the body frame. Because the measurements may be made by physically distinct instruments, $\boldsymbol{G}$ is not required to be either orthogonal or normal. $G$, therefore, contains 9 independent parameters. Together with the 3 independent

\footnotetext{
* This work was supported by the National Aeronautics and Space Administration/Goddard Space Flight Center, Greenbelt, MD, USA, Contract GS-35F-4381G, Task Order no. S-32415-G.

${ }^{\dagger}$ This paper is declared a work of the U.S. Government and is not subject to copyright protection in the United States.
}

biases, the complete calibration requires the determination of 12 parameters.

The on-orbit gyro calibration methods described in this paper intend to determine the 3 elements of $\bar{b}$ and the 9 independent elements of $G$ based on on-orbit measurements.

During normal mission activities spacecraft angular rates are usually maintained approximately constant by the control system. Under these conditions of nearly constant rates, and if gyro biases as well as attitudes are included in an attitude determination method, the attitude accuracy depends only weakly on the accuracy of $\boldsymbol{G}$. This is a consequence of the fact that at constant rates the off diagonal elements of $\boldsymbol{G}$ and errors in the diagonal elements produce constant contributions to $\omega$ which can be modeled as biases. The existence of a bias vector that can compensate for errors in the G-matrix is shown as follows.

A condition that a batch least-square or Kalman filter attitude solution be accurate over a significant time span is that the effective estimated rates be consistent with sensor observations. Thus, if an erroneous Gmatrix is used to compute rates from gyro observations, a necessary condition for an accurate solution (and small sensor residuals) is that the overall gyro rates must be approximately equal to the true gyro rates. If the true G-matrix and bias vector are represented by $\boldsymbol{G}$ and $\bar{b}$, and an initial estimate of the Gmatrix is represented by $\Gamma$, then for any period with observed rates, $\bar{\omega}_{o}$, the necessary condition for an accurate attitude solution is that there exist some solved-for bias, $\vec{\beta}$, such that:

$$
G\left(\vec{\omega}_{o}-\vec{b}\right)=\Gamma\left(\vec{\omega}_{o}-\vec{\beta}\right)
$$

Solving for $\vec{\beta}$ as a function of $\omega_{0}$ gives:

$$
\vec{\beta}\left(\omega_{o}\right)=\vec{\omega}_{o}-\Gamma^{-1} G\left(\vec{\omega}_{o}-\vec{b}\right)
$$

1 
Since $\Gamma, \boldsymbol{G}$, and $\bar{b}$ are constant, $\bar{\beta}$ can only be constant if $\bar{\omega}_{o}$ is also constant. Conversely, if the rates are constant, a bias vector can be found that will fit the data by compensating for errors in the assumed $\mathrm{G}$ matrix.

Even though attitude accuracy during constant-rate periods is not affected by gyro calibration, there are three reasons that calibration is desirable:

- Gyro calibration improves targeting accuracy in maneuvers

- Gyro calibration improves attitude accuracy when the rates are not constant

- Gyro calibration reduces drift during contingencies if the spacecraft attitude is determined from gyro propagation without sensor update.

In practice accurate gyro calibration can only be performed using data from periods with different distinct rate profiles. Since at constant rates errors in $\boldsymbol{G}$ and $\vec{b}$ are not separable, data from either periods with different but constant rates or from several maneuvers must be used to unambiguously determine the parameters. A minimum of 4 distinct rate profiles must be used to provide observability of the 12 independent gyro parameters.

The need for different rate environments makes it difficult to calibrate gyros using normal mission data. Usually, special maneuvers must be planned to insure complete observability. Since these maneuvers are often quite different from normal mission activities, it is often difficult to design them while maintaining mission health and safety constraints. Because repetition of gyro calibration maneuvers presents an unusual burden on operations, it is important that the calibration algorithm be accurate and robust. In an operations environment, methods that are easy to use, that require less user intervention, and that can make use of normal-operations maneuvers, are also desirable.

In more than 10 years of calibrating gyros for the NASA's Goddard Space Flight Center (GSFC), we have experience with four algorithms for calibrating gyros. The major portion of this paper contains descriptions of these four algorithms: The Davenport algorithm, the Delta-bias algorithm, the Filtersmoother algorithm, and a Batch Least-squares IRU Calibration (BICal) algorithm. BICal is a new algorithm and this paper includes a description and evaluation of its performance during testing.
BICal has the advantages of being no less accurate than the other methods (and potentially more accurate), of making complete, optimum use of all of the data, of significantly reducing the restrictions on calibration maneuver design and scheduling, and of greatly reducing the operator intervention needed for calibration.

We describe each algorithm and our experience using it, concentrating on its accuracy, robustness, and ease of use.

The underlying assumption made in all of the gyro calibration algorithms described below is that over a significant period, the G-matrix and biases remain constant. Experience has shown the G-matrix is stable over a period of years'. The bias is known to drift, but, depending on the stability of the gyros, can be considered stable over a period of weeks or months ${ }^{2}$.

For all of the algorithms described with the exception of BICal, the data used for calibration is assumed to be obtained from periods separated by no more than a few days (or at most weeks) in order to insure that the solved-for biases can be considered constant over the data range. BICal, since it has the capability of solving for separate biases for each interval of data used, can employ data spans separated by longer times.

\section{ALGORITHMS}

\section{Davenport Algorithm}

\section{Description}

The Davenport algorithm takes advantage of the fact that accurate attitudes can be obtained for periods of constant rates, even if the G-matrix and bias are not well known. These attitudes are based on observations from the most accurate sensors available, as well as rate data. As described above, accurate attitudes may be attained by absorbing any errors in the Gmatrix into an effective bias. The Davenport algorithm minimizes differences between these accurate attitudes and attitudes propagated from other accurate attitudes. In order to obtain complete observability the rates in the propagation intervals must be linearly independent and span the rotation space. In order to span the space a complete solution requires at least 4 such intervals.

Let a series of accurate attitudes obtained before and after each interval be denoted $q_{1 b}, q_{2 b} \cdots q_{n b}$ and $q_{1 a}, q_{2 a} \cdots q_{n a}$. Separate estimates of the attitudes 
can be obtained by propagating the gyro measurements through the interval:

$$
q_{i a}^{e s t}=P_{i, a \leftarrow b} q_{i b}
$$

where $\boldsymbol{P}$ is, of course, a function of the gyro parameters and the observed rates, and propagates the attitude before the interval, $i$, into an estimated attitude after the interval. The effective loss function for the Davenport algorithm is constructed from quaternion products of the accurate and the estimated attitudes summed over the independent intervals.

The solution for $G$ and $\bar{b}$ is obtained by minimizing the loss function. Details are given elsewhere ${ }^{3}$.

A useful variation on the Davenport algorithm is particularly applicable to on-board systems ${ }^{4}$. This method relies on the on-board computer to determine the quaternion after a maneuver. If the maneuver control uses gyros only, the control system stops the maneuver at an attitude found by propagation of the pre-maneuver attitude using the gyros. When accurate attitude sensor data is reintroduced into the control system, the attitude settles to the true target attitude. The magnitude of the settling is equivalent to the quaternion products used to construct the Davenport algorithm loss function.

\section{Experience}

The Davenport algorithm has been used as the normal operational algorithm for GSFC ground calibration. It has proved accurate and robust in the calibration of the Upper Atmosphere Research Satellite (UARS), the Extreme Ultraviolet Explorer (EUVE), the Total Ozone Mapping Spectrometer-Earth Probe (TOMSEP), the Rossi X-ray Timing Experiment (RXTE), the Submillimeter Wave Astronomy Satellite (SWAS), the Tropical Rainfall Measurement Mission (TRMM), and the Earth Observing System -AMI (Terra).

In all of these missions the calibration results were evaluated by measuring the decrease in maneuver targeting errors when on-orbit calibration parameters were used in place of prelaunch values.

For UARS there was a limitation on the accuracy of the results due to mission restrictions on calibration maneuver design and due to software limitations on the processing of gyro telemetry. Several recalibrations have been performed on the UARS gyros giving more accurate, and generally consistent results. These recalibrations have shown that the gyro alignments and scale factors are stable over periods of years (after initial postlaunch changes).
For RXTE, gyro data dropout during the acceleration period of the calibration maneuvers caused errors. These errors would have been reduced had the gyro data been telemetered as accumulated angles rather than rates.

\section{Delta-Bias Algorithm}

\section{Description}

If attitudes and effective gyro biases are determined during several periods with constant but different rates, the complete set of gyro parameters can be determined from the effective gyro biases.

Data from several periods of nearly constant observed rate of $\vec{\omega}_{1}, \bar{\omega}_{2} \ldots \bar{\omega}_{n}$, are adjusted using an assumed bias, $\vec{\beta}$, and G-matrix, $\Gamma$ to give adjusted rates, $\vec{w}_{1}, \vec{w}_{2} \ldots \vec{w}_{n}$. This $\vec{\beta}$ is not the solution of Eq. (3) that gives the exact rate so an attitude determination method can compensate by solving for an additional bias for each period, $\bar{\beta}_{1}, \bar{\beta}_{2} \ldots \bar{\beta}_{n}$. An estimate of the true bias $\vec{b}$ and true G-matrix, $\boldsymbol{G}$, can be determined as follows. The true rates in the body frame can be expressed as:

$$
G\left(\vec{\omega}_{i}-\vec{b}\right)=\Gamma\left(\vec{\omega}_{i}-\vec{\beta}\right)-\vec{\beta}_{i} \equiv \vec{w}_{i}-\vec{\beta}_{i}
$$

Defining the true parameters in term of changes from the assumed parameters:

$$
\begin{aligned}
& \vec{b} \equiv \vec{\beta}+\Delta \vec{\beta} \\
& G \equiv(I+\Delta G) \Gamma
\end{aligned}
$$

and rearranging yields:

$$
\begin{gathered}
\Gamma\left(\vec{\omega}_{i}-\vec{\beta}\right)-\vec{\beta}_{i}=(I+\Delta G) \Gamma\left(\vec{\omega}_{i}-\vec{\beta}-\Delta \vec{\beta}\right) \\
\bar{\omega}_{i}-\vec{\beta}-\Gamma^{-1} \vec{\beta}_{i}=\left(I+\Gamma^{-1} \Delta G \Gamma\right)\left(\vec{\omega}_{i}-\vec{\beta}-\Delta \vec{\beta}\right)
\end{gathered}
$$

Assuming the corrections are small and using the definition of $\vec{w}_{i}$, this can be rearranged to:

$$
\Gamma^{-1} \vec{\beta}_{i} \cong \Delta \vec{\beta}-\Gamma^{-1} \Delta G \vec{w}_{i}
$$

or

$$
\vec{\beta}_{i} \cong \Gamma \Delta \vec{\beta}-\Delta G \vec{w}_{i}
$$

For 4 independent sets of rates, Eq. (10) can be solved for $\Delta \boldsymbol{G}$ and $\Delta \bar{\beta}$ and therefore $G$ and $\vec{b}$. If more than 
4 sets are available, a least-squares estimate of $\boldsymbol{G}$ and $\vec{b}$ can be obtained.

The effective loss function for the Delta-bias algorithm is:

$$
L_{\text {delta bias }}=\sum_{i}\left(\Gamma \Delta \vec{\beta}_{\text {estimated }}-\Delta G_{\text {estimated }} \vec{w}_{i}-\beta_{i}\right)^{2}
$$

\section{Experience}

The Delta-bias algorithm has been used on UARS, RXTE, and Terra as a comparison and check on the Davenport algorithm. In each of these cases it provided results consistent with those from the Davenport algorithm. For UARS and RXTE ${ }^{5}$ it avoided the periods of unusable or unavailable data during maneuvers and since it uses data during nearly constant rate periods was less sensitive to data dropout.

It has also been recently used on data from Terra for a direct comparison with other gyro calibration algorithms. The results of its use on Terra data, and the comparison with the other methods are given below.

\section{Filter-Smoother Algorithm}

\section{Description}

The Filter-smoother algorithm was developed to reduce the effort needed for calibration using the Davenport algorithm. Common Extended Kalman Filters (EKFs) used in attitude determination and control solve for attitude and gyro bias. The EKF equations can be derived from the minimization of the trace of the state error covariance matrix.

The gyro-calibration Filter-smoother has an augmented state function including the G-matrix elements and also includes a smoother step. The smoother step follows the filter by rerunning the filter backwards and combining the results of the forward and backwards filters. For each time, the two results are averaged, weighted by the inverses of their covariances.

\section{Experience}

The Filter-smoother algorithm was developed for RXTE and run using data from calibration maneuvers. The results were unsatisfactory. After the start of the first maneuver the filter quickly diverged and produced physically unreasonable values (biases and scale factors that would have corresponded to multiple $2 \pi$ radian rotations of the spacecraft between adjacent gyro observations).
Numerous attempts were made to diagnose this problem, but none were successful.

- If the filter was initialized with parameters very near the true solution (found using the Davenport algorithm) the filter converged, but any significant deviation of initial parameters from ideal resulted in divergence.

- Many attempts at tuning the filter by adjusting the sensor uncertainty, the initial parameter uncertainty, and the gyro noise estimates proved unsuccessful in promoting convergence.

- The input data was closely examined and manually "repaired" without improving success.

- The software code was closely examined but no errors were found.

The final conclusion was that although, in principal, the Filter-smoother Algorithm should give accurate results, either it has an unacceptably small radius of convergence, or this implementation of the algorithm was faulty in some undetected way.

\section{BICal Algorithm}

\section{Description}

The BICal algorithm was developed as an extension of a Batch Least-squares (BLS) attitude determination algorithm that has been used at GSFC for many years. In the BLS algorithm the attitude at an epoch and gyro bias are determined that minimize sensor residuals over the entire batch of data.

In the familiar Wahba loss function ${ }^{6}$

$$
L_{\text {Wahba }}=\sum_{j} W_{j}\left(A \hat{R}_{j}-\hat{O}_{j}\right)^{2}
$$

the sum is over all observations by all sensors. $A$, the attitude to be estimated, is a rotation matrix that rotates inertial frame vectors to the body frame. $O_{j}$ is the $\boldsymbol{J}^{\text {th }}$ observation vector in body coordinates, $\boldsymbol{R}_{j}$ is the corresponding reference vector in inertial coordinates and $\boldsymbol{W}_{j}$ is a weight for the observation chosen so that the loss function minimum corresponds to the minimum state covariance.

If the observations occur at different times, the attitude, $A$, must be replaced by the attitude at the time of the measurement, $A_{t}$. The attitude at any time can be estimated as an epoch attitude, $\boldsymbol{A}_{0}$, propagated to the time:

$$
A_{t}=P_{t \leftarrow 0} A_{0}
$$


where the propagation uses the gyro data and, in the BLS method, is treated as an explicit function of the gyro bias (assumed to be constant over the batch). The BLS method thus finds the epoch attitude and bias vector that minimize the loss function:

$$
L_{B L S}=\sum_{j} W_{j}\left(P_{t \leftarrow 0}(\omega, \vec{b}) A_{0} \hat{R}_{j}-\hat{O}_{j}\right)^{2}
$$

BICal extends the BLS method in three ways:

- BICal allows data from multiple independent time periods to be used together

- It assumes each time period to have a separate epoch attitude, allows different time periods to have different biases, and assumes a common G-Matrix for all time periods.

- It explicitly solves for the various epoch attitudes and biases as well as for the elements of the Gmatrix to minimize the loss function:

$$
L_{B I C a l}=\sum_{i} \sum_{j} W_{j i}\left(P_{t \leftarrow 0 i}\left(\omega, \vec{\beta}_{k_{i}}, G\right) A_{0 i} \hat{R}_{j i}-\hat{O}_{j i}\right)^{2}
$$

where the index $i$ indicates the time period containing the data and $\boldsymbol{k}_{\boldsymbol{i}}$ indicates the bias to be applied during the $i^{\text {th }}$ time interval. Because the same bias may be solved for over more than one time period, the range of $k_{i}$ must be less than or equal to that of $i$.

\section{Experience}

The BICal algorithm is a newly developed algorithm and has been tested using data from only two missions. The results of its use on the Terra mission are given below. It has also been used with data from the Wide-Field Infrared Explorer (WIRE).

The WIRE data used consisted of normal mission data taken over a period of 9 hours in 4 discontinuous intervals. WIRE underwent numerous maneuvers in this time as shown by the reported gyro rates, presented in Figure 1.

The maneuvers in the first and fourth interval were each sufficiently varied to provide complete observability of all gyro calibration parameters. Because the WIRE gyros had a large bias drift, the bias in the fourth interval differed substantially from that in the first or of the first three taken together.

The gyro and sensor data were used in BICal to solve for attitudes, gyro biases, and G-matrixes. If data from the fourth interval were included in the solution a separate bias had to (and was) accurately determined for this interval.

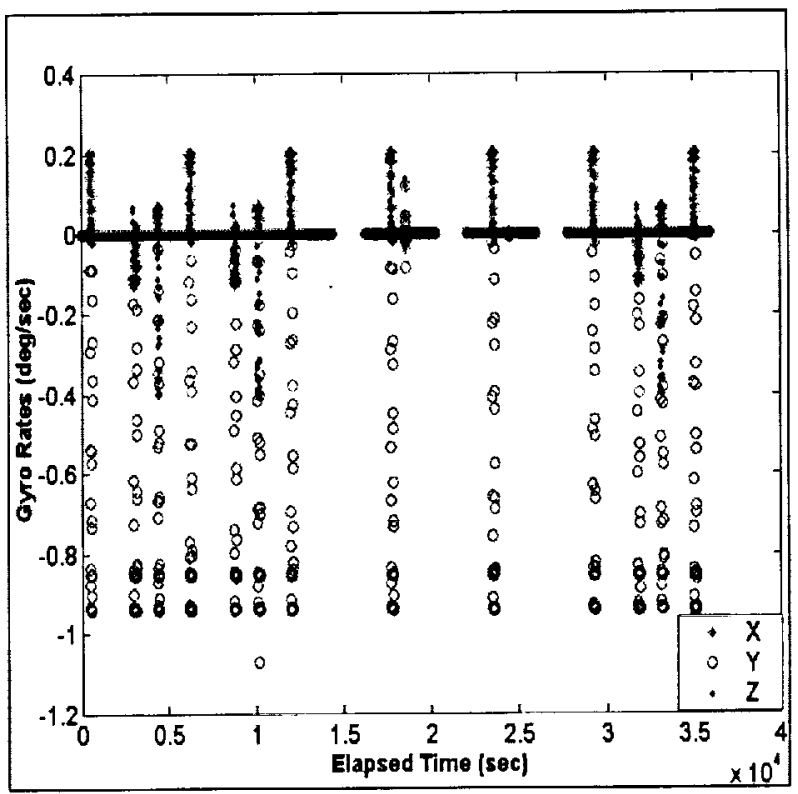

Fig. 1 WIRE Gyro Rates During Test Period

Once the biases and G-matrix were determined, the gyro data were readjusted using these parameters. The gyro data were then corrupted by introduction of known additional biases and G-matrix. To test BICal, it was executed many times using randomly corrupted gyro data to determine if it converged on the known additional biases and G-matrix.

BICal returned virtually identical correct epoch attitudes, gyro biases, and G-matrix under even extreme conditions. Convergence was attained with initial attitude errors of tens of degrees, initial G-matrix elements in error by several tenths, and initial bias errors of tenths of degrees per hour.

Additional investigations were made of the effect of noise on the accuracy of the results. In Monte Carlo calculations of the effect of gyro noise on results, 80 BICal runs were performed with additional gyro noise, generated with a normal distribution, a zero mean and a standard deviation of $10^{-6} \mathrm{rad} / \mathrm{sec}^{3 / 2}$ (about an order of magnitude larger than the specified values for good gyros). Statistics on the determined biases, G-matrix elements, and star tracker residuals after propagation are shown in Table 1.

A similar Monte Carlo calculation was performed using 114 samples with the star tracker noise increased randomly in each with a normal distribution and standard deviation of 60 arcsec. These results are 
also shown in Table 1. These results show that BICal is insensitive to sensor and gyro noise as well as robust with respect to starting conditions.

Table 1. Monte Carlo Results: Errors in BICal Parameters With Increased Input Noise

\begin{tabular}{|c|c|c|}
\hline 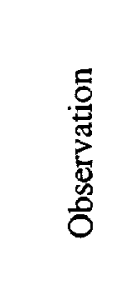 & 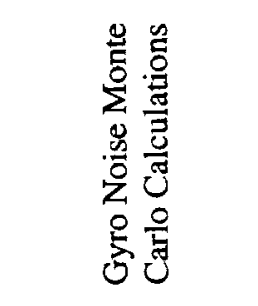 & 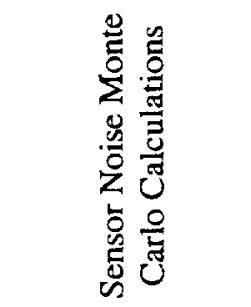 \\
\hline$\sigma_{\text {bias }}$ & $3.02 \times 10^{-5} \mathrm{deg} / \mathrm{hr}$ & $3.87 \times 10^{-5} \mathrm{deg} / \mathrm{hr}$ \\
\hline$\sigma_{\Delta \mathrm{G}}$ & $3.24 \times 10^{-5}$ & $3.27 \times 10^{-5}$ \\
\hline$\sigma_{\text {Residual }}$ & $11.1 \operatorname{arcsec}$ & 40.1 arcsec \\
\hline
\end{tabular}

The values in Table 1 represent mean standard deviations. For the bias, the standard deviation over the ensemble of cases was computed for each axis and the mean of these three values is shown. For the Gmatrix the standard deviations over the ensemble of cases was computed of the differences between the G-matrix and the identity matrix and the mean over the 9 elements is presented. For star tracker residuals the standard deviation of the residuals in each run is averaged over the ensemble, and the averages of these values over the three axes is presented.

\section{COMPARISON OF ALGORITHMS}

\section{Error Analysis}

It is useful to compare the expected accuracies of the four calibration algorithms discussed in this paper. The full problem of determining the covariance matrix for all 12 calibration parameters is too unwieldy to solve explicitly. However one can still gain insight by studying a simplified system. In a universe with only two space dimensions, the attitude reduces to a single rotation angle, and gyro calibration consists of estimating only a bias and a scale factor correction. This 3-parameter state vector is a good model for studying the errors on a single IRU axis; it only neglects the misalignments of the sensitive axes, and experience has shown that the effect of misalignment error is usually smaller than that of scale factor error.

The approach used in the Davenport method is to estimate the attitude at selected epoch times. The differences between these attitudes are compared with the attitude changes obtained by integrating the gyro rates. The discrepancies in these attitudes are used to solve for the bias and scale factor correction. In the error analysis presented here, the uncertainties in the epoch attitudes are the source of error in the estimated bias and scale factor. Similarly, in the Delta-bias method, the uncertainties in the epoch biases lead to the errors in the bias and scale factor.

A single attitude maneuver is adequate to separate the effects of bias and scale factor; however, for symmetry, this analysis assumes a positive and a negative slew with the covariance matrix calculated at the midpoint during the zero-rate period between slews. This eliminates the correlation between bias and scale factor and makes the comparisons simpler.

The scenario for the analysis is that the measured rotation rate is zero for a time, $T$, during which $N$ sensor observations are made. Then there is a slew through an angle $\Theta$ taking a time $T_{\text {slew. }}$. This is followed by another zero-rate period with duration $T$ containing $N$ sensor observations, a slew of $-\Theta$ back to the initial attitude, again taking time $T_{\text {slew }}$, and a final zero-rate period with duration $T$ and containing $N$ sensor observations. An epoch attitude and bias are estimated for each of the three zero-rate periods. Process noise is neglected in the analysis.

Since the rates are constant and an effective bias is solved for, the errors in the three epoch attitudes are due only to random sensor noise and are independent of any systematic gyro calibration error. Each epoch is taken at the midpoint of the corresponding time span $T$ which makes the uncertainties smallest and makes the correlation between attitude and bias zero. Also, the attitude errors are uncorrelated since the three observation periods do not overlap. Since process noise is neglected in this model (no random gyro error), each attitude uncertainty is simply $\sigma_{o b s} / N^{1 / 2}$ where $\sigma_{o b s}$ is the standard deviation of the sensor error. Similarly, the bias uncertainty for each epoch can be shown to be $2 \sqrt{3} \sigma_{o b s} /\left(T N^{1 / 2}\right)$ to order $1 / N^{2}$. 
Table 2. Parameter Uncertainties

\begin{tabular}{|c|c|c|}
\hline & $\sigma_{\text {bias }}$ & $\sigma_{\text {scale }}$ \\
\hline 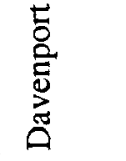 & $\frac{\sigma_{o b s}}{\sqrt{2 N}\left(T+T_{\text {slew }}\right)}$ & $\sqrt{\frac{3}{2}} \frac{\sigma_{o b s}}{N^{1 / 2} \Theta}$ \\
\hline 䍘 & $\frac{2 \sigma_{o b s}}{\sqrt{3 N}}\left(\frac{1}{T^{2}}+\frac{2}{T_{s l e w}^{2}}\right)^{1 / 2}$ & $2 \sqrt{\frac{3}{2}} \frac{\sigma_{o b s}}{N^{1 / 2} \Theta}$ \\
\hline 离 & $\frac{\sigma_{o b s}}{\sqrt{2 N\left[\left(T+T_{\text {slew }}\right)^{2}+T^{2} / 8\right]}}$ & $\sqrt{\frac{3}{2}} \frac{\sigma_{o b s}}{N^{1 / 2} \Theta}$ \\
\hline
\end{tabular}

Carrying out the analysis for the Davenport algorithm, the first and second epoch attitudes are propagated to the times of the second and third using the measured rates. The discrepancies in these attitudes are algebraically related to the required bias and scale factor corrections. The covariance of the bias and scale factor is a congruent transformation of the covariance of the two attitude discrepancies. Note that the two attitude discrepancies have one epoch attitude in common, so there is a correlation between them that must be included in their covariance matrix. The resulting Davenport calibration uncertainties are given in Table 2. It may be possible to improve the bias accuracy slightly by choosing epoch times away from the midpoints or by subdividing the zero-rate periods. In practice, it is difficult to choose the best epoch times given sensor data of varying accuracy and irregular spacing. The arbitrariness in choosing the epoch times is one of the weaknesses of the Davenport method as implemented.

The Delta-bias method uses the effective biases from constant rate intervals to solve for the calibration parameters; the method does not make use of the attitude discrepancies obtained by integrating the gyro rates as in the Davenport method, so the Delta-bias method is expected to be less accurate. The algorithm requires bias estimates during at least two periods with different constant rates so a slightly modified scenario was used. The modified scenario starts with a constant rate maneuver by an angle $\Theta$ of duration $T_{\text {slew }}$ containing $N$ sensor observations. This is followed by a zero-rate period of duration $T$ containing $N$ sensor observations and finally another constant rate slew of $-\Theta$ with duration $T_{\text {slew }}$ and containing $N$ sensor observations. This scenario maintains the same total number of observations as the original scenario, however the total time span is less.

The true bias and scale factor are written as a function of the effective biases for the three constant rate periods. Since there are three equations for the two unknowns, the bias and scale factor are obtained using a pseudo-inverse of the coefficient matrix. The effective biases have uncorrelated uncertainties as given above. The covariance matrix for the bias and scale factor is a transformation of the covariance of the effective biases. The resulting Delta-bias calibration uncertainties are given in Table 2.

As long as process noise is not included, the error covariance matrix will be the same for BICal as for the Filter-smoother method since both methods make optimal use of the information input in the context of the model. (Process noise here means random gyro errors, as opposed to the systematic bias and scale factor errors being solved for.) The uncertainties were determined for a Filter-smoother but apply equally to BICal.

For the Filter-smoother method, we use the same scenario as for the Davenport method. The covariance matrix can be propagated and updated using the standard Kalman filter algorithm. The covariance was initialized with large values on the diagonal to represent a lack of initial knowledge about the state. It was propagated and updated to the midpoint. A backward Kalman filter was initialized at the last point and propagated and updated back to the midpoint. The covariances from the forward and backwards filters are combined to obtain the smoothed covariance. After a certain amount of algebra, the uncertainties shown in Table 2 for the Filter-smoother (or BICal) were obtained.

If process noise is included, the Filter-smoother will yield somewhat larger estimated uncertainties, and these will be a more accurate reflection of reality. This would make the filter more correctly "optimal" than a batch algorithm without process noise.

Table 2 shows that the uncertainties of the bias and scale factors computed using all of the methods share similar dependencies on sensor noise, number of observations, time span, and slew angle. The BICal (or Filter-smoother) method has the lowest uncertainties, but the difference with the Davenport method is small and normally negligible. The Delta-bias method has somewhat larger uncertainties than the other methods. This has been borne out by our experience with flight 
data as discussed below. We have found it to be a useful back-up procedure that provides quick corroboration of the more accurate methods. In the real world, all of these methods are complicated by the effects of misalignments of the gyro axes especially for Earth-oriented spacecraft where the projection of a large pitch rate onto the other axes can be significant. Also, as discussed previously, the Filter-smoother did not perform well with RXTE data, but this analysis shows that at least in theory it should be the most accurate method.

In practice, BICal or Filter-smoother will often do better than Davenport by using observations during slews; the Davenport method cannot use this data since it must avoid acceleration periods in order to have accurate epoch attitudes. However, the most important advantages of BICal are not concerned with accuracies, but involve ease of use and applicability to widely separated data spans, as discussed in the Conclusions section.

\section{Analysis of Flight Data}

A direct comparison of the Davenport Algorithm, the Delta-bias Algorithm, and BICal was accomplished using data from Terra. Terra calibration gyro maneuvers were performed in a period of about 6 hours on April 13, 2000. Between maneuvers, sufficient time at constant rates was allowed to permit accurate attitudes to be determined using data from Terra's 2 Ball CT-601 star trackers and the gyros.

The gyro data used in the calibration were in the form of accumulated angles, sampled every eight seconds. Every eight seconds each star tracker reports the position of up to 5 stars. A total of 11566 star observations in tracker 1 and of 11413 star observations in tracker 2 were identified by ground software in the calibration data.

The eight calibration maneuvers started with a positive 10 degree roll followed by a roll back to nominal. This sequence was repeated in the negative roll direction. There were similar 30 deg positive and negative yaw offset maneuvers. Terra is an Earth-oriented spacecraft and at all times, it maintained its 1 rotation per orbit attitude motion about orbit normal. Because of this constant rotation, the offset attitudes resulted in changes of the pitch rates by the cosine of the offset angle and therefore provided pitch observability. The gyro rates during the entire period used for calibration are shown in Figure 2.

The Davenport algorithm, the Delta-bias algorithm and BICal were all used to calibrate the gyros and gave reassuringly similar results. Differences in corresponding G-matrix elements derived by different methods were no larger than about $10^{-4}$ (corresponding to about 20 arcsec in alignment difference), and differences in the biases, no larger than about $0.01 \mathrm{deg} / \mathrm{hr}$.

When each of the three sets of gyro parameters were used to readjust the data and a batch least-squares attitude solution was computed over the calibration span, small differences in the quality of the calibration parameters became evident. The epoch of the attitude determination was chosen at the start of the data, so that once the attitude was found, it could be propagated through all of the maneuvers using the gyro parameters (including the solved-for bias as an additional adjustment). The epoch attitudes were very similar with the difference between the BICal and Davenport solutions less than an arcsec and between these solutions and the Delta-bias solution about 5 arcsec.

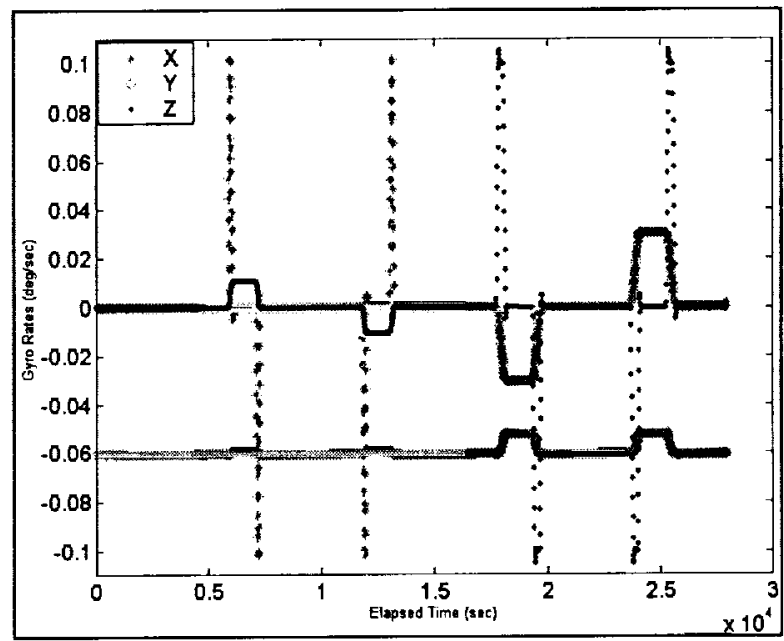

Fig. 2 Terra Gyro Rates During Calibration Period

Table 3 shows the standard deviation of the star tracker residuals as well as the additional bias needed to minimize the residuals. For comparison, the case with prelaunch gyro parameters (labeled "no calibration" in Figure 3) is also included. Note that outlier sensor observations differing by more than 3 standard deviations from the means have been discarded.

All three of the calibrations improve the residuals markedly. Among the calibrations, the BICal and Davenport algorithms are essentially identical and both have very small residuals. Since the loss function of the attitude batch least-squares runs that produced these results is identical to that used in BICal 
(although the state vector in BICal includes the Gmatrix), it is hardly surprising that the biases are essentially zero. Figure 3 shows the residuals of these attitude determinations as a function of time. It is clear that the BICal and Davenport algorithms give somewhat improved results compared to the Deltabias algorithm and that all results are much superior to precalibration values.

Table 3. Comparison of Terra Gyro Calibration Results

\begin{tabular}{|c|c|c|c|c|}
\hline \\
\hline & 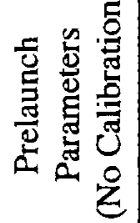 & 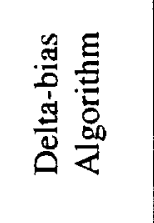 & 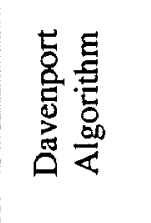 & $\frac{\pi}{\mathscr{U}}$ \\
\hline$\sigma_{x 1}(\operatorname{arcsec})$ & 50.48 & 5.61 & 3.36 & 3.28 \\
\hline$\sigma_{y 1}(\operatorname{arcsec})$ & 52.91 & 5.21 & 3.21 & 3.02 \\
\hline$\overline{\sigma_{21}(\operatorname{arcsec})}$ & 53.29 & 5.91 & 3.64 & 3.38 \\
\hline$\sigma_{\mathrm{x} 2}(\operatorname{arcsec})$ & 50.89 & 5.46 & 3.16 & 3.06 \\
\hline$\sigma_{\mathrm{y} 2}(\operatorname{arcsec})$ & 52.14 & 6.77 & 4.10 & 3.92 \\
\hline$\sigma_{z 2}(\operatorname{arcsec})$ & 52.55 & 6.98 & 4.78 & 4.37 \\
\hline$\Delta \beta_{\mathrm{x}}(\mathrm{deg} / \mathrm{hr})$ & 0.0933 & -0.000162 & 0.00169 & 0.00004 \\
\hline$\Delta \beta_{\mathrm{y}}(\mathrm{deg} / \mathrm{hr})$ & 0.4463 & -0.000094 & -0.00011 & 0.00011 \\
\hline$\Delta \beta_{2}(\mathrm{deg} / \mathrm{hr})$ & -0.2977 & -0.000047 & -0.00058 & -0.00007 \\
\hline
\end{tabular}

Differences in the parameter sets obtained can also be seen in Figures 3 and 4. Figure 3 shows star tracker residuals obtained using different calibration parameters during the $+30 \mathrm{deg}$ yaw maneuver and the corresponding $-30 \mathrm{deg}$ yaw maneuver in the same data used for Table 3. For each parameter set, batch least-squares attitudes were computed using gyro data previously adjusted with the selected parameters. Attitudes at different times are the epoch attitude, propagated using the gyro data to each time. For each sensor observation the root-sum-square (RSS) of the three axes is taken and the residual value represented in the figure is the mean of the RSSs of all star tracker observations at a time. The residuals found using BICal parameters (indicated by stars in the figure) are virtually identical to those found using the Davenport parameters (indicated by squares). The residuals found using the Delta-bias parameters are also similar before the first maneuver but increase somewhat with each maneuver. The residuals found using the prelaunch parameter values (labeled "No calibration" and indicated by $\mathrm{x}$ ) are uniformly high. Because BLS methods compute an epoch attitude and then propagate it, and the epoch chosen here was before the maneuvers, the fact that the sensor residuals for the BI$\mathrm{Cal}$ and Davenport cases are as small after maneuvers as near epoch, demonstrates that the error in gyro propagation is negligible.

During maneuvers the residuals obtained using any of the parameter sets were noticeably larger, especially during the acceleration portion of the maneuver. The errors in the acceleration portion arise from modeling the spacecraft rates as constant during the eight second period between gyro measurements. Additional errors due to inaccurate time tags, for example, may also contribute to these errors. Figure 4 shows the differences between some of the attitudes computed for Figure 3. The attitude differences are shown through all 8 maneuvers. Attitudes computed using the BICal and Davenport parameters are virtually identical and their differences are not shown. The differences shown use the BICal parameter base attitude as a reference and, in addition to attitudes derived using the Delta-bias and prelaunch parameters, attitudes computed by the onboard computer (OBC) Kalman filter are included.

The Delta-bias parameters result in propagation errors that increase with each maneuver but the increase is no more than about 10 arcsec for any maneuver. The corresponding propagation errors using prelaunch parameters reach as much as 50 arcsec. In addition, errors in the gyro biases cause an attitude drift of as much as 120 arcsec during the periods in which the spacecraft is flying in an offset attitude.

The OBC attitudes differ only slightly from those found with BICal parameters at the start of the data. After each maneuver, however, the OBC attitude error increases, and during the offset attitude periods drifts because star tracker observations are not used to update the on-board filter. After the use of star tracker observations is resumed (at nominal attitude) the attitude errors return to small values. The error due to this drift was as much a 250 arcsec over the 8 minute yaw offset period. This implies that Terra, without the more accurate gyro calibration parameters would drift by about $0.5 \mathrm{deg} / \mathrm{hr}$ if controlled by gyros alone. 


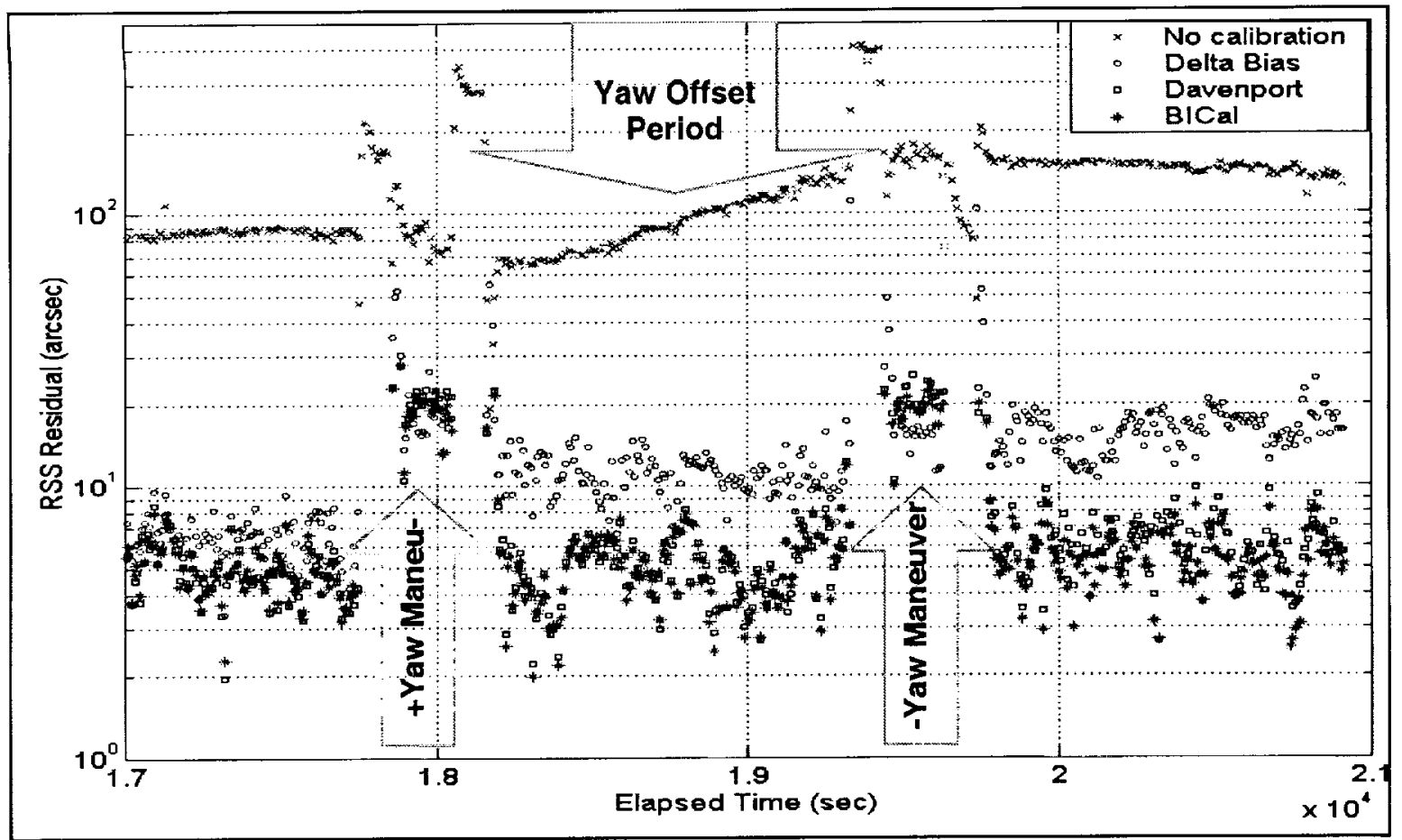

Fig. 3 Star Tracker Residuals With Different Calibration Parameters

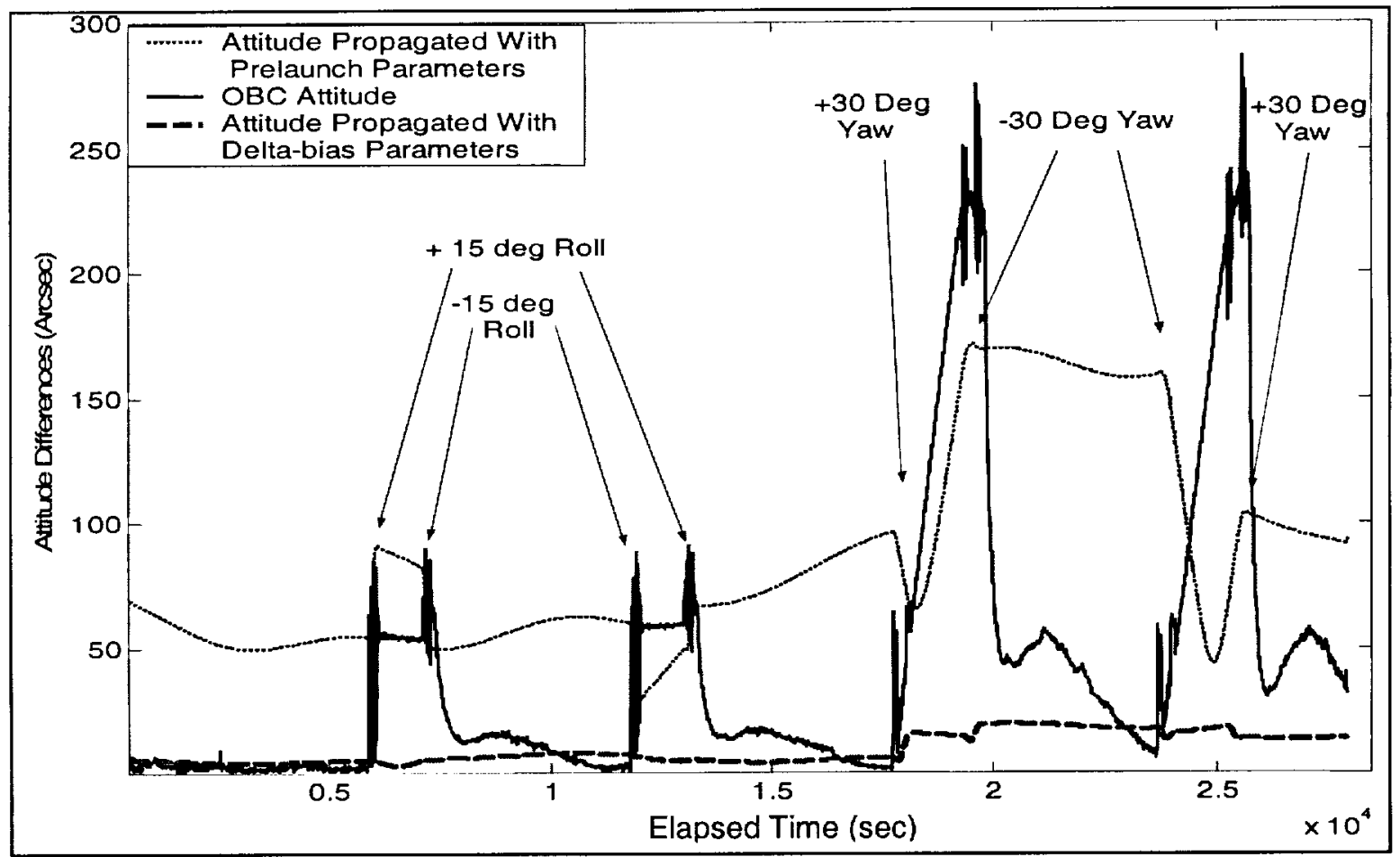

Fig. 4 Attitude Differences Between BLS Attitudes Based on BICal Parameters and Others

10

American Institute of Aeronautics and Astronautics 


\section{CONCLUSIONS}

When it is necessary to calibrate satellite gyros after launch there are several reliable algorithms that can be used. The Delta-bias algorithm gives slightly less accurate results than do the Davenport and BICal algorithms but this accuracy difference is small enough not to make any practical difference.

Because the inherent accuracy of the algorithms is so similar, the major factors influencing choice of algorithm are differences in ease of use and impact on mission operations.

The Delta-bias algorithm requires at least 4 periods during which rates are different, linearly independent, and nearly constant. This condition seldom arises during normal mission operations so in order to use this algorithm, periods must be set aside from normal operations during which the rates must differ from nominal. Design of such periods, which must be long enough to provide sufficient sensor observations for determination of accurate attitudes and effective biases, is often difficult.

The Davenport and BICal algorithms can, in principal, often use normal mission maneuvers for at least part of the data needed for calibration. For several reasons, $\mathrm{BICal}$ is expected to be the more useful and used of these algorithms. The advantages of BICal over the Davenport algorithm include:

- The BICal algorithm uses sensor data during maneuvers, providing greater observability.

- BICal can be used with normal mission data for missions (such as SWAS and TOPEX/Poseidon) in which constant rate periods are not normal.

- BICal is less demanding on calibration maneuver design because constant rate periods do not have to be scheduled at the ends of maneuvers.

- BICal can be used in cases where maneuvers are separated by such long times that the gyro biases during the different maneuvers are different. Therefore,

- BICal requires less operator intervention and is less labor intensive to use. In the Davenport algorithm, adjusted data must be examined to find the intervals with constant rate at the start and end time of calibration intervals. Separate attitudes must be computed for each of these termini, after which the algorithm processing can be performed. The BICal algorithm is used directly on adjusted data with no additional processing needed.

\section{ACKNOWLEDGEMENTS}

The authors wish to thank Dr. Gary Welter for very helpful discussions.

\section{REFERENCES}

1- W. Davis, J. Hashmall, J. Garrick, and R. Harman, "Post-launch Calibration of Spacecraft Attitude Instruments", Spaceflight Dynamics 1993, Part II, AAS93-325 (1993).

2- J. Sedlak, J. Hashmall, and V. Airapetian, "Comparison of On-orbit Performance of Rate Sensing Gyroscopes", to appear in Spaceflight Dynamics 2000, Biarritz, France, (2000)

3- J. Keat, "Gyro Calibration Analysis for the High Energy Astronomy Observatory-A (HEAO-A)", Computer Sciences Corporation, CSC/TM-77/6082 (1977)

4- G. Welter, J. Boia, M. Gakenheimer, E. Kimmer, and D. Channell, "Variations on the Davenport Gyroscope Calibration Algorithm", Proceedings of the Flight Mechanics/Estimation Theory Symposium, NASA CP-3333, Goddard Spaceflight Center, Greenbelt, Maryland, (1996)

5- D. Fink, W. Davis, et. al., "X-Ray Timing Explorer (RXTE) Postlaunch Report", Computer Sciences Corporation, GSFC 553-FDD-96/005R0UD0 (1996)

6- L. Markley, and D. Mortari, "How to Estimate Attitude From Vector Observations", Proceedings of AAS/AIAA Astrodynamics Specialist Conference, AAS 99-427, Girdwood, Alaska, USA, (1999) 